(Figure 1). No significant heterogeneity was detected between studies $(P=0.25$, $P^{2}=23.0 \%$ ). The data from the longitudinal studies investigating the association between smoking and spinal radiographic progression is still been assessed.

Conclusions: The preliminary results of our meta-analysis showed that smoking is associated with increased cumulative spinal structural damage in patients with AS. Rheumatologists should encourage AS patients to quit smoking.

Disclosure of Interest: None declared

DOI: 10.1136/annrheumdis-2017-eular.4659

\section{OP0121 VALIDATION OF MRI STRUCTURAL LESIONS USING COMPUTED TOMOGRAPHY IN PATIENTS WITH AXIAL SPONDYLOARTHRITIS}

W.P. Maksymowych ${ }^{1,2}$, D. Loeuille ${ }^{3}$, M. Raynal ${ }^{4}$, J. Melchior ${ }^{5}$, M.-A. D'Agostino ${ }^{6}$, J. Paschke ${ }^{2}$, R.G. Lambert ${ }^{7} .{ }^{1}$ Medicine, University of Alberta; ${ }^{2}$ CaRE Arthritis, Edmonton, Canada: ${ }^{3}$ Rheumatology, CHRU de Nancy;

${ }^{4}$ Rheumatology, CHRU de Nancy, Vandouvre-lès-Nancy; ${ }^{5}$ Rheumatology, CHRU de Nancy, Vandoeuvre les Nancy; ${ }^{6}$ Rheumatology, Université Paris Ouest, Paris, France; ${ }^{7}$ Radiology, University of Alberta, Edmonton, Canada

Background: MRI can detect both inflammatory and structural lesions in the sacroiliac joints (SIJ) of patients with axial SpA. However, standard MRI sequences do not directly depict bone and the appearance of erosion may vary according to the presence/absence of inflammation. Consequently, further validation using an accepted gold standard, namely, computed tomography (CT), is essential.

Objectives: We aimed to assess the detection of structural lesions in the SIJ by MRI using CT as the gold standard.

Methods: CT scans from 44 patients (26 females, mean age 49.4 years, mean symptom duration 9.1 years) were reconstructed in the semicoronal plane parallel to the superior border of the sacrum, as for conventional MRI evaluation of the SIJ, and scoring of lesions by CT was confined to this plane. Structural lesions were scored in consecutive slices in SIJ quadrants (erosion, sclerosis) or SIJ halves (ankylosis) on a dichotomous basis (present/absent) using the same anatomical principles for defining SIJ quadrants as developed for the SPARCC MRI SIJ inflammation and structural scores. T1W MRI scans of the SIJ from the same cases conducted at the same time as CT were assessed independently for structural lesions (erosion, fat, backfill, ankylosis, sclerosis) blinded to CT assessments using the SPARCC method. Agreement between CT and MRI was assessed by kappa statistics. Sensitivity/specificity of MRI for CT lesions was calculated. The primary analysis was based on lesions detected concordantly by both readers at the level of the individual iliac/sacral joint surface (erosion, sclerosis) or the individual joint (ankylosis, backfill).

Results: With CT as gold standard and a lesion defined as being present when recorded in the same location on at least 1 coronal slice by both readers, MRI-defined ankylosis had $56.3 \%$ sensitivity and $100 \%$ specificity for CT ankylosis (Table). For erosion, sensitivity and specificity of MRI was $81.3 \%$ and $96.2 \%$, and for sclerosis, sensitivity and specificity of MRI was $50 \%$ and $97 \%$, respectively. Agreement between CT and MRI for erosion increased when the cut-off for presence of a lesion was set at 2 slices but only marginally for sclerosis, even when the cut-off for presence of a lesion was set at 3 slices. Lesions observed on CT corresponding to backfill on MRI were ankylosis, erosion, and sclerosis, in $66.7 \%, 66.7 \%$, and $80 \%$ of backfill lesions, respectively.

\begin{tabular}{|c|c|c|c|c|c|}
\hline \multicolumn{2}{|c|}{ Concordance for 2 readers } & \multirow[t]{2}{*}{ Type of Lesion } & \multirow[t]{2}{*}{$\mathrm{K}$ value } & \multirow[t]{2}{*}{ Sensitivity of MRI } & \multirow[t]{2}{*}{ Specificity of MR } \\
\hline$\underline{\text { MRI lesion + }}$ & CT lesion + & & & & \\
\hline \multirow[t]{3}{*}{$\geq 1$ slice } & $\geq 1$ slice & Ankylosis & 0.67 & $56.3 \%$ & $100 \%$ \\
\hline & & Erosion & 0.76 & $81.3 \%$ & $96.2 \%$ \\
\hline & & Sclerosis & 0.47 & $50.0 \%$ & $97 \%$ \\
\hline \multirow{3}{*}{$\geq 2$ slices } & $\geq 2$ slices & Ankylosis & 0.71 & $60.0 \%$ & $100 \%$ \\
\hline & & Erosion & 0.83 & $84.6 \%$ & $98.1 \%$ \\
\hline & & Sclerosis & 0.53 & $42.9 \%$ & $99.2 \%$ \\
\hline \multirow[t]{3}{*}{$\geq 2$ slices } & $\geq 3$ slices & Ankylosis & 0.83 & $75.0 \%$ & $100 \%$ \\
\hline & & Erosion & 0.84 & $90 \%$ & $98.1 \%$ \\
\hline & & Sclerosis & 0.53 & $42.9 \%$ & $99.2 \%$ \\
\hline
\end{tabular}

Conclusions: Ankylosis and erosion on MRI correspond closely with the same type of lesion observed on CT. Both new bone formation and erosion are frequently evident on CT at locations where backfill is observed on MRI supporting the hypothesis that backfill is an intermediary tissue between erosion and ankylosis.

Disclosure of Interest: None declared

DOI: 10.1136/annrheumdis-2017-eular.5427

\section{THURSDAY, 15 JUNE 2017 \\ Clinical and therapeutical news in systemic sclerosis}

\section{OP0122 VENOUS THROMBOEMBOLISM IN SYSTEMIC SCLEROSIS} PREVALENCE, RISK FACTORS AND IMPACT ON SURVIVAL
Toronto, Toronto, Canada; ${ }^{2}$ King Fahad Central Hospital, Jizan, Saudi Arabia; ${ }^{3}$ Anesthesia, University of Toronto, Toronto, Canada

Background: Systemic sclerosis (SSc) is characterized endothelial dysfunction and vasculopathy, which may result in thrombosis. Venous thromboembolism (VTE) is a vascular phenomenon that includes deep vein thrombosis (DVT) and pulmonary embolism (PE).

Objectives: To evaluate the epidemiology of VTE in SSc, specifically cumulative incidence, risk factors and impact of VTE on survival.

Methods: We conducted a cohort study of patients who fulfilled the ACR-EULAR classification criteria for SSc attending the Toronto Scleroderma Program 19702017. DVT was defined as the presence of thrombus on doppler ultrasound of either upper or lower extremity. PE was defined as the presence of thrombus on CT angiogram of the thorax. Time to all-cause mortality was evaluated in Kaplan Meier and Cox models.

Results: 1181 subjects $(971(82 \%)$ female, $210(18 \%)$ male) were included. There were $40(3.4 \%)$ VTE events. The cumulative incidence of VTE was 2.7 $(95 \% \mathrm{Cl} 1.9,3.7)$ per 1000 patient years. The presence of ILD, PAH, ScL70 antibody, anticardiolipin antibody, coronary artery disease, diabetes mellitus and PVD occurred more frequently in subjects who developed VTE.

Table 1. Venous Thromboembolism in SSc

\begin{tabular}{lccc}
\hline Characteristics & VTE $(\mathrm{N}=40)$ & No VTE $(\mathrm{N}=1141)$ & Relative Risk $(95 \% \mathrm{Cl})$ \\
\hline Diffuse subtype & $12(30 \%)$ & $382(33 \%)$ & $1.04(0.84,1.30)$ \\
Male sex & $9(23 \%)$ & $201(18 \%)$ & $1.28(0.71,2.30)$ \\
Raynaud's phenomenon & $39(98 \%)$ & $1087(95 \%)$ & $1.02(0.97,1.08)$ \\
Esophageal dysmotility & $35(88 \%)$ & $981(86 \%)$ & $1.02(0.90,1.15)$ \\
Interstitial lung disease & $23(58 \%)$ & $393(34 \%)$ & $1.67(1.26,2.20)^{\star}$ \\
Pulmonary arterial hypertension & $28(70 \%)$ & $367(32 \%)$ & $2.18(1.75,2.71)^{\star}$ \\
Abnormal nailfold capillaries & $14(35 \%)$ & $354(31 \%)$ & $1.13(0.73,1.74)$ \\
Digital ulcers & $13(33 \%)$ & $386(34 \%)$ & $0.96(0.61,1.51)$ \\
Scl 70 antibody & $11(28 \%)$ & $186(16 \%)$ & $1.73(1.03,2.91)^{\star}$ \\
Anticentromere antibody & $5(13 \%)$ & $215(19 \%)$ & $0.68(0.30,1.56)$ \\
Anticardiolipin & $3(8 \%)$ & $14(1 \%)$ & $6.27(1.88,20.9)^{\star}$ \\
Coronary artery disease & $9(23 \%)$ & $98(9 \%)$ & $2.69(1.47,4.92)^{\star}$ \\
Hypertension & $11(28 \%)$ & $237(21 \%)$ & $1.32(0.79,2.22)$ \\
Diabetes mellitus & $6(15 \%)$ & $61(5 \%)$ & $2.81(1.29,6.10)^{\star}$ \\
Hyperlipidemia & $5(13 \%)$ & $78(7 \%)$ & $1.83(0.78,4.27)$ \\
Peripheral vascular disease & $8(20 \%)$ & $41(4 \%)$ & $5.57(2.79,11.08)^{\star}$ \\
Cancer & $7(8 \%)$ & $127(11 \%)$ & $1.57(0.79,3.14)$ \\
Atrial fibrillation & $6(15 \%)$ & $45(4 \%)$ & $3.80(1.72,8.39)^{\star}$ \\
\hline
\end{tabular}

${ }^{*}$ Denotes statistical significance.

On multivariate logistic regression PAH (OR $3.77(96 \% \mathrm{Cl} 1.83$, 8.17), ScL70 antibodies (OR $2.4595 \% \mathrm{Cl} 1.07,5.30$ ), anticardiolipin antibodies (OR 5.70 $(95 \% \mathrm{Cl} 1.16,21.2)$ and PVD (OR $5.31(95 \% \mathrm{Cl} 1.99,12.92)$ were independent predictors of VTE. Subjects with ILD more frequently experienced DVT (RR 2.85 $95 \% \mathrm{Cl}(1.08,7.54)$ but not PE (RR $1.82(95 \% \mathrm{Cl} 0.89,3.70)$. There were 440 deaths. There was no significant difference in survival between those with and without VTE (HR $1.6(95 \% \mathrm{Cl} 0.70,1.92)$. Only the presence of ILD (HR 1.54 $(95 \% \mathrm{Cl} 1.27,1.88)$ or PAH (HR $1.35(95 \% \mathrm{Cl} 1.10,1.65)$ remained independent predictors of mortality.

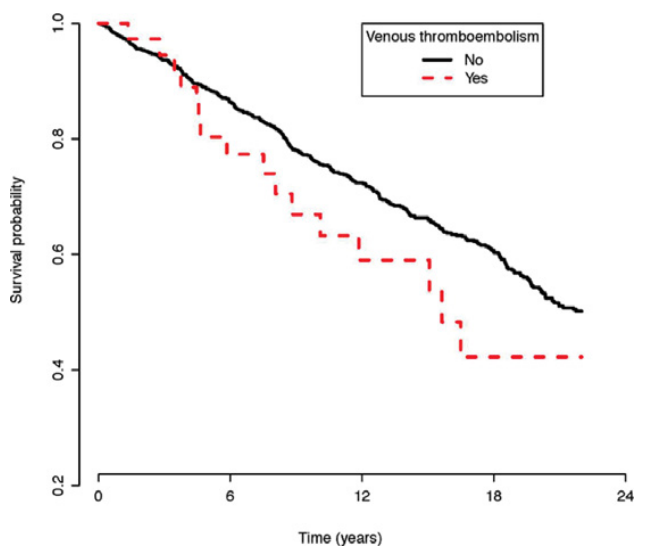

Figure 1. Kaplan Meier survival curves of SSc subjects with and without venous thromboembolism. Log rank test $\mathrm{p}=0.54$

Conclusions: The risk of VTE in SSc is not increased, as the incidence of VTE in SSc is comparable to the general population. The presence of PAH, PVD, ScL70 and anticardiolipin antibodies are risk factors for VTE in SSc. VTE does not independently affect SSc survival.

Acknowledgements: Dr. Johnson is supported by the Canadian Institutes of Health Research.

Disclosure of Interest: None declared

DOI: 10.1136/annrheumdis-2017-eular.5629 